\title{
A RARE CASE OF HYPERCALCAEMIA: DOUBLE TROUBLE WITH A TWIST
}

Rebecca Sagar ${ }^{1}$, Afroze Abbas ${ }^{1}$

${ }^{1}$ Leeds Centre for Diabetes and Endocrinology, St James' University Hospital, Leeds Teaching Hospitals, Leeds, LS9 7TF, UK

\section{Hyperparathyroidism (2014)}

- 60 year old gentleman presented in 2014 with slurred speech, confusion and cerebellar signs.

- Elevated calcium (2.9mmol/L) and PTH (14.9pmol/L).

- Family History: Hyperparathyroid-jaw tumour syndrome (HPT-JT) affecting daughter and son (germline Leu63Pro missense mutation in CDC73 exon 2).

- MRI brain: Cerebellar lesions in the peridentate regions (right larger than left).

- Positive voltage-gated potassium channels

- Neurology opinion: Cerebellar lesions and positive voltage-gated potassium channels, both of indeterminate aetiology.
A

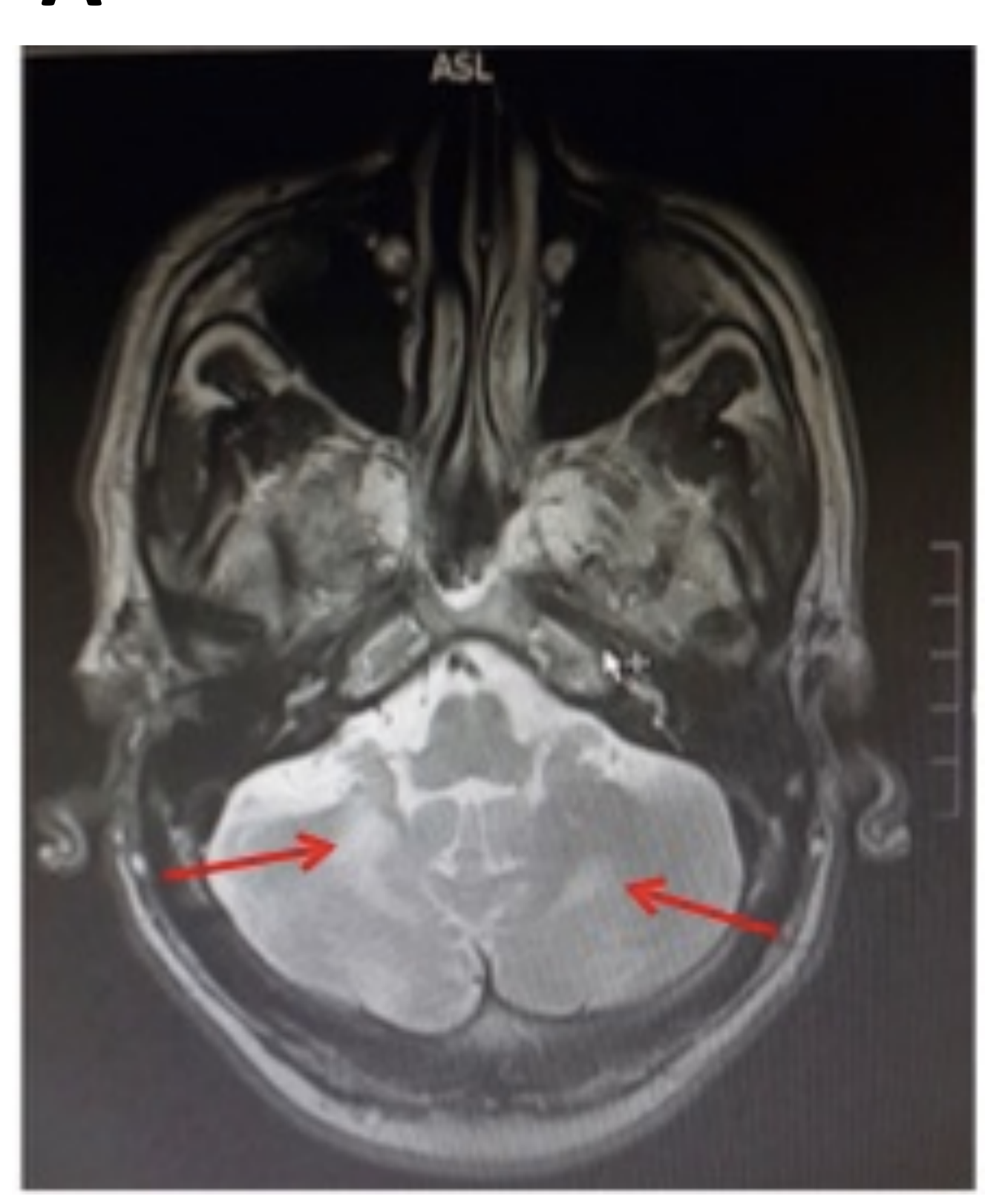

B

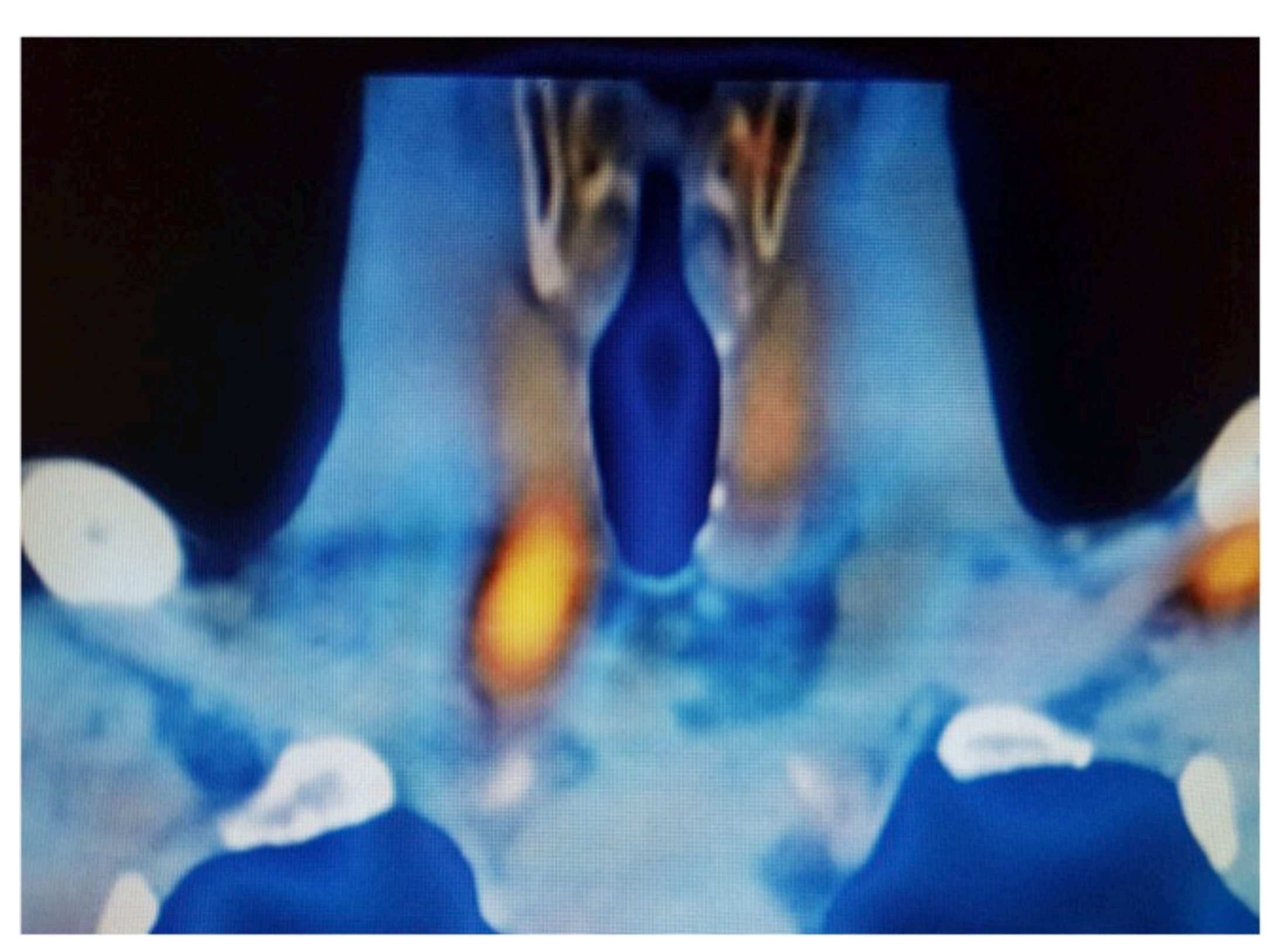

FIGURE 1: A) MRI Brain showing peridentate lesions (arrows) and B) CT SPECT demonstrating a right inferior parathyroid lesion

- Serum ACE: $60.0 \mathrm{u} / \mathrm{L}$ (8-52).

- CT TAP: Normal, specifically no evidence of malignancy or lymphadenopathy.

- CT SPECT: Right inferior adenoma

- Minimally invasive parathyroidectomy performed

- Post-op: Normocalcaemic, with normal PTH.

\section{Progress (2015-2016)}

- Regular outpatient review: Normocalcaemia for 2.5 years following parathyroidectomy. Systemically well.

- No evidence of tumours associated with HPT-JT.

- Serial MRI scans: Partial resolution of cerebellar findings.

References:

${ }^{1}$ Coexisting Primary Hyperparathyroidism and Sarcoidosis Cause Increased Angiotensin-Converting Enzyme and Decreased Parathyroid Hormone and Phosphate Levels. V Lim, BL Clarke; The Journal of Clinical Endocrinology \& Metabolism ,2013. Vol 98 (5): 1939-1945

\section{Sarcoidosis (2017)}

- In April 2017, 2 month history non-specifically unwell, tired and weight loss. Calcium $2.31 \mathrm{mmol} / \mathrm{L}$, PTH 0.6 $\mathrm{pmol} / \mathrm{L}$, Vitamin $\mathrm{D}<20 \mathrm{nmol} / \mathrm{L}$.

- Investigations planned, however given Vitamin D supplementation 40,000 Units per week for 7 weeks

- 2 weeks later admitted acutely unwell with calcium $3.71 \mathrm{mmol} / \mathrm{L}, \mathrm{PTH}<0.3 \mathrm{pmol} / \mathrm{L}$.

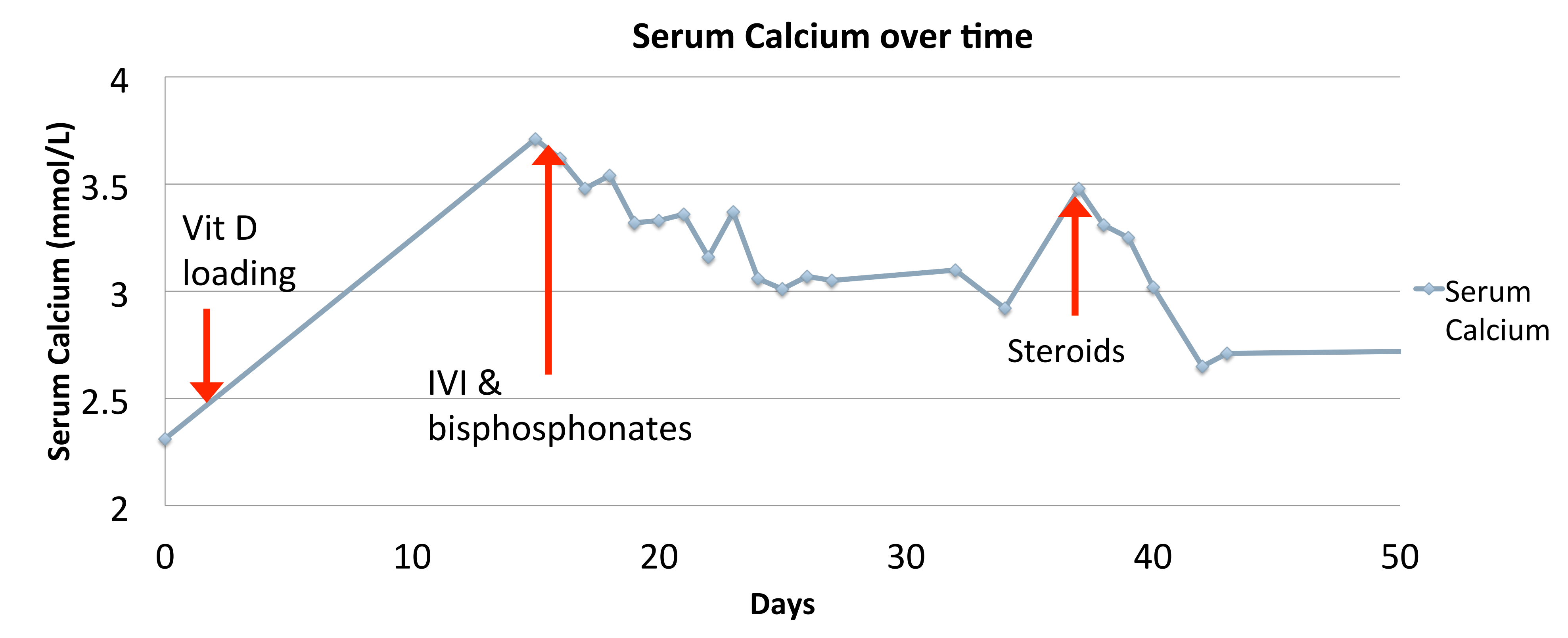

FIGURE 2: Serum calcium over time including interventions (arrows)

\begin{tabular}{|l|l|l|}
\hline TEST & RESULT & RANGE \\
\hline Serum ACE & $88.8 \mathrm{u} / \mathrm{L}$ & $20-70 \mathrm{u} / \mathrm{L}$ \\
\hline 1,25 di-hydroxyvitamin D3 & $>250 \mathrm{pmol} / \mathrm{L}$ & $20-120 \mathrm{pmol} / \mathrm{L}$ \\
\hline PTHrP & $<1.0 \mathrm{pmol} / \mathrm{L}$ & $0.7-1.8 \mathrm{pmol} / \mathrm{L}$ \\
\hline
\end{tabular}

- CT TAP: extensive lymphadenopathy, splenomegaly and lung changes. Differentials were lymphoma or sarcoidosis. No evidence of parathyroid tumour recurrence.

- FNA biopsy of lymph nodes: granulomatous inflammation, confirming a diagnosis of sarcoidosis.

\section{Conclusion}

This is an unusual case of a gentleman with hypercalcaemia due to HPT-JT syndrome, successfully treated by parathyroidectomy, only for him to then suffer with recurrent hypercalcaemia, precipitated by vitamin D3 supplementation, in the context of undiagnosed sarcoidosis.

Cases of primary hyperparathyroidism and sarcoidosis presenting concurrently have been previously reported $^{1}$. However, this is the first reported case of sarcoidosis associated with HPT-JT syndrome.

The nature of his bilateral cerebellar lesions remain unresolved. 